\section{§27. Generation of Toroidal Current Sheet at Sawtooth Crash}

Itoh, $\mathrm{K}$.

Itoh, S.-I. (RIAM, Kyushu Univ.)

Soltwisch, H. (Univ. Bochum)

Koslowski, H. R. (IPP, Julich)

The mechanism to generate the toroidal sheet current after the sawtooth crash (observation on TEXTOR tokamak [1]) is discussed. A possible hypothesis is presented. The crash of central pressure causes the steep gradient near the boundary between the flattened region and the unperturbed region. This sharp pressure gradient gives rise to the strong secondary current (Pfirsch-Schluter current). The location, polarity, magnitude and time evolution of the current sheet are analyzed. Comparison with present experimental data is made, and future necessary measurements are discussed.

The intrinsic feature of the sawtooth crash is that the crash time is much shorter than the energy confinement time, and that the region of the drastic collapse is localized in the center. Owing to these characteristics, the steep pressure gradient established at the end of the crash $r \simeq r_{K A M}$. (See Fig.1.) By use of the diffusion coefficient, $\chi_{L}$, the layer thickness $\Delta$ is evaluated as

$$
\Delta \simeq \sqrt{\chi_{L} \tau_{c r}} .
$$

The steep pressure gradient is estimated as

$$
\left|\frac{d p}{d r}\right| \simeq \frac{p(0)-p_{f}}{r_{1}} \sqrt{\frac{\chi_{M}}{\chi_{L}}}
$$

By use of this pressure gradient, the Pfirsch-Schluter current is calculated. This current is mainly in the toroidal direction and has the in-out asymmetry. It is given as

$$
J_{\|}=(1+2 q) \sqrt{\frac{\chi_{M}}{\chi_{L}}} \frac{p(0)-p_{f}}{B r_{1}} \cos \theta .
$$

This result indicates that there will appear a localized toroidal current just after the crash of the central pressure. The direction of this induced current is in the same direction as the main plasma current in the outside of the torus $(\theta \simeq 0)$ and is opposite to the plasma current at the inside $(\theta \simeq \pi)$. It is more convenient to compare, with experiments, the magnitude of the current driven by the sharp pressure gradient.
The total current in the current sheet, $I_{s}$, is estimated as

$$
I_{s} \simeq 6 r_{K A M} \frac{p(0)-p_{f}}{B} .
$$

The sheet current disappears as the steep pressure gradient diffuses away. The typical time scale for the decay of the localized current is given as the heat-pulse-propagation time to the point of the interest.

The prediction by the model is compared to the experimental observation on the polarity , radial thickness, magnitude, poloidal structure and temporal dynamics. The hypothesis agrees with several aspects of the observation and is not clearly rejected. However, there are several signs that the improvement of the model is necessary, i.e., poloidal structure. In order to test more precisely, further possible experimental tests are studied.

This work is performed under the JapanTEXTOR collaboration programme. This work is partly supported by the Grant-in-Aid for Scientific Research of MoE Japan, by the collaboration programme of NIFS and by the collaboration programme of Advanced research Center of Kyushu University.

\section{Reference}

[1] Soltwisch H, Fuchs G, Koslowski H R, Schlueter J, Waidmann G 1991 Proc. 18th Eur. Conf. on Controlled Fusion and Plasma Physics, Berlin, Europhys. Conf. Abstr., Vol.15C, PartII, 17 (Eur. Phys. Soc. Geneva).



Fig.1 Profiles of the pressure after (solid line) and before (dashed line) of the crash. 